\title{
Microbiome in Rheumatoid Arthritis and Celiac Disease: A Friend or Foe
}

\author{
Kawther Elsouri ${ }^{1}$, Vania Arboleda ${ }^{1}$, Samantha Heiser ${ }^{2}$, Marc M. Kesselman ${ }^{3}$, Michelle Demory Beckler ${ }^{4}$
}

1. Medicine, Nova Southeastern University Dr. Kiran C. Patel College of Osteopathic Medicine, Fort Lauderdale, USA 2. Medicine, Nova Southeastern University Dr. Kiran C. Patel College of Allopathic Medicine, Fort Lauderdale, USA 3. Rheumatology, Nova Southeastern University Dr. Kiran C. Patel College of Osteopathic Medicine, Davie, USA 4. Microbiology and Immunology, Nova Southeastern University Dr. Kiran C. Patel College of Allopathic Medicine, Fort Lauderdale, USA

Corresponding author: Kawther Elsouri, ke552@mynsu.nova.edu

\begin{abstract}
Rheumatoid arthritis (RA) and celiac disease (CD) are both autoimmune diseases with increasing global prevalence. These two diseases have been connected based on similar HLA mutations, serological markers, rheumatological, and gastrointestinal manifestations. In this review, we discuss the role of the oral and gut microbiome in the development and progression of RA and CD. Here, we highlight similar microbial dysbiosis and how these alterations in composition can lead to worsening disease severity in both CD and RA. Additionally, we analyze the role of probiotics in regulating the microbiome and improving symptoms associated with RA and CD.
\end{abstract}

Categories: Allergy/Immunology, Rheumatology

Keywords: celiac disease, probiotics, gut-joint axis, oral microbiome, gut microbiome, rheumatoid arthritis, autoimmune diseases

\section{Introduction And Background}

Rheumatoid arthritis (RA) is a chronic systemic autoimmune disease that can manifest clinically as symmetrical joint damage, limited range of motion, and swelling [1]. RA primarily affects the lining of the synovial joints, leading to progressive disability, socioeconomic burdens, and possibly premature death. Poorly controlled or severe cases have a higher risk for extra-articular manifestations: cardiovascular illness, keratitis, pulmonary granulomas/rheumatoid nodules, pericarditis/pleuritis, small vessel vasculitis, and other non-specific symptoms [1]. Within four weeks of gastrointestinal or genitourinary bacterial infections or viral infections, acute or chronic forms of arthritis can arise, which can mimic seropositive RA [2].

There are two major subtypes of RA depending on the presence or absence of anti-citrullinated protein antibodies (ACPAs) [1]. Both subtypes present with joint pain, swelling, and limited range of motion.

Received 03/26/2021 Review began 05/08/2021 Review ended 06/08/2021 Published 06/09/2021

\section{(c) Copyright 2021}

Elsouri et al. This is an open access article distributed under the terms of the Creative Commons Attribution License CC-BY 4.0., which permits unrestricted use, distribution, and reproduction in any medium, provided the original author and source are credited.
However, seropositive RA patients, where serum ACPA is present, tend to have more severe manifestations and phenotypes, including higher levels of pain, inflammation, and bone destruction than seronegative RA patients. ACPAs, present in approximately $67 \%$ of patients, can be found long before the onset of the joint symptoms [1]. The genetic risk factor associated with ACPA-positive RA is found in the human leukocyte antigen (HLA)-DR gene. The two alleles HLA-DR1 and HLA-DR4 have "shared epitopes" (SEs) [3], and are considered primary risk factors for RA outcomes due to their role in the production of ACPAs [4]. In addition to genetic risk factors, mucosal sites exposed to a high load of bacterial antigens may represent the initial site of autoimmune generation [4]. Emerging data have implicated the microbiome as an extra-articular trigger of RA pathogenesis [4,5].

Following a proper diagnosis, the treatment options for RA include long-term disease-modifying antirheumatic drugs (DMARDs, including biologic agents) and intermittent short courses of corticosteroids, which aim to maintain quality of life [6]. The problem with RA treatments is the severity of the known adverse effects of DMARDs which include liver and GI effects, drug-induced lupus erythematosus, Clostridium difficile colitis, and infertility [6].

Celiac disease (CD) is an autoimmune disease whereby its pathogenesis is triggered by the consumption of gluten and gluten components, including wheat, barley, and rye [7]. Clinical manifestations of CD can include villous atrophy of the small intestine, malnutrition, vitamin deficiency, osteoporosis, anemia, chronic diarrhea, cancer, fertility problems, and psychiatric and behavior abnormalities [7]. Similar to RA, microbial and viral infections have been associated with CD development in genetically predisposed patients $[7]$.

$\mathrm{CD}$ is diagnosed by villous atrophy on duodenal biopsy and can also include the presence of serum antibodies targeting tissue transglutaminase (tTg) and immunogenic gluten proteins/peptide sequences [8]. In patients with $\mathrm{CD}$, $\mathrm{tTg}$ cleaves gluten and produces glutenins and gliadins (33-mer peptide fragment, found 
in wheat, rye, and barley) which act as highly immunogenic epitopes that have a linked affinity for the protein products of HLA DR3-DQ2 and/or DR4-DQ8 alleles [9]. Moreover, greater than 99\% of individuals with CD have HLA DR3-DQ2 and/or DR4-DQ8 alleles [7]. While anti-tTg antibodies are used as a diagnostic tool, the pathogenic mechanism is thought to be T cell-mediated. Patients with active CD tend to exhibit similar levels of CRP and pro-inflammatory cytokines as RA patients due to inflammation to intestinal damage.

Preservation of the intestinal villi is greatly improved with early diagnosis of $\mathrm{CD}$, however difficulty in determining the correct diagnosis limits early detection due to a growing percentage of clinical manifestations being extraintestinal without any gastrointestinal symptoms at all [9]. Currently, treatment plans center around a gluten-free diet (GFD) to control the immune responses observed in CD. However, a diet modification is not effective for all $\mathrm{CD}$ patients due to continued, but inadvertent ingestion of gluten, commonly from gluten contamination of products presumed to be gluten-free [10].

\section{Review}

RA and CD share multiple autoimmune pathogenic features and clinical manifestations. The presence of anti-gluten antibodies is more prevalent in RA patients than controls [10], and a similar increase in the presence of tTg auto-antibodies was found in RA [11]. The incidence of CD has increased significantly over the last six decades and an analogous trend can be observed for rheumatic diseases in females and aging populations [7]. Many studies have suggested a gut-joint axis, whereby inflammation in the gut mucosa precedes joint manifestations, up to years in advance, suggesting underlying immune mechanisms and the gastrointestinal environment can influence the onset and progression of joint disease (Figure 1) [7]. A casual link between the onset and progression of RA and CD has been linked. Both have similar clinical presentations, diagnostic features, epidemiological trends, and $\mathrm{CD}$ involves chronic autoimmune inflammation of the GI tract (Table 1). To this end, we hypothesize that CD and RA are linked. A major factor that draws a bridge between the two is the gut-joint axis, whereby events occurring in the gut can impact the joints. We sought to understand how dysbiosis of the gut and oral microbiomes as components of the gut-joint axis link CD and RA. Below, we identify specific strains and species involved in the microbiome dysbiosis seen in RA and CD patients and their role in the development and progression of both autoimmune diseases. Additionally, we identify how probiotics may play a role in decreasing disease severity. 


\section{Cureus}

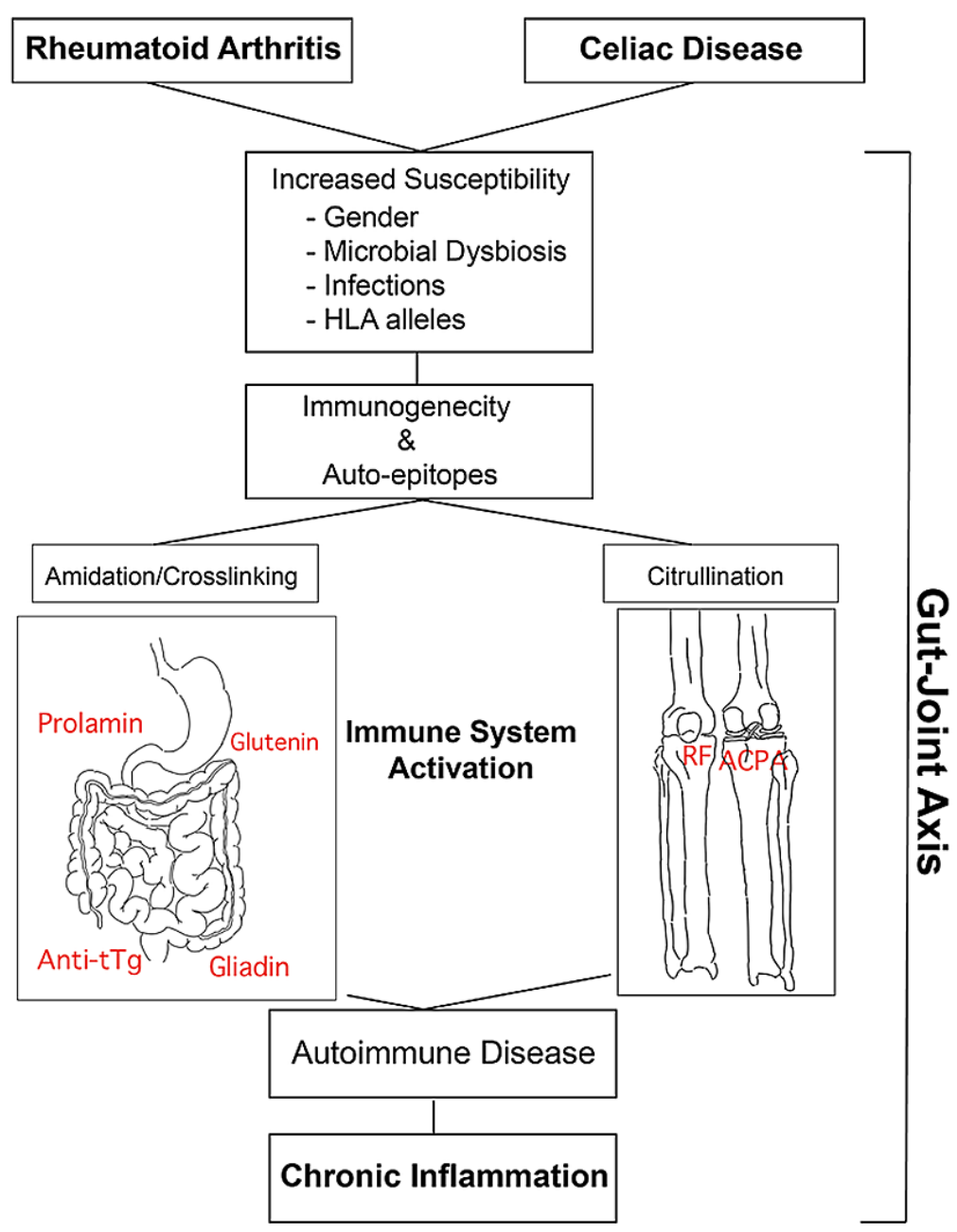

FIGURE 1: Diagram depiction of the gut-joint axis.

HLA: human leukocyte antigen; anti-tTg: anti-tissue transglutaminase antibodies; ACPA: anti-citrullinated protein antibodies; RF: rheumatoid factor 


\section{Cureus}

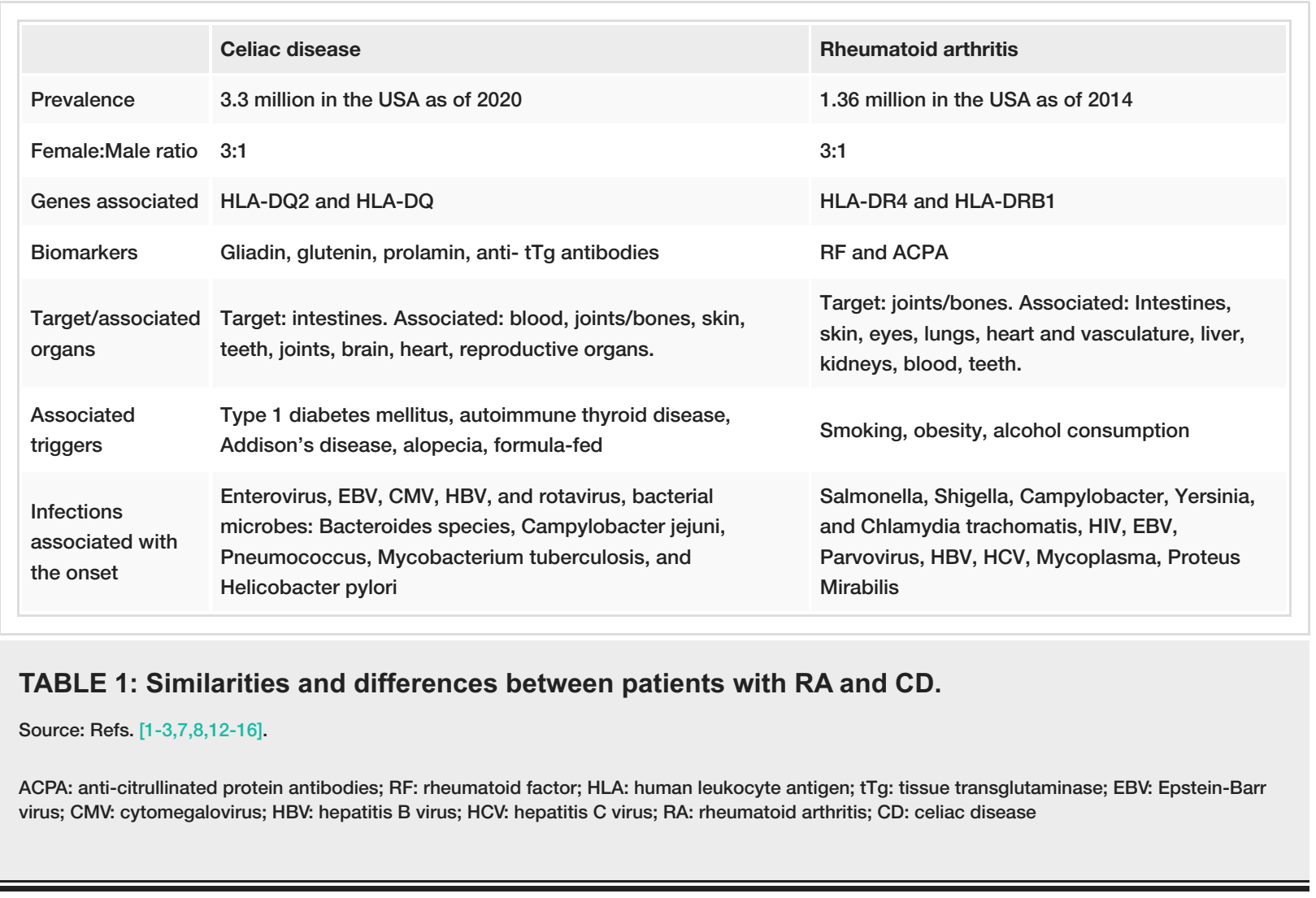

\section{Role of oral and gut microbiomes in RA}

The oral cavity harbors over 700 species of bacteria that contribute to the health and physiological status of the oral cavity [17]. The oral microbiome is extremely dynamic due to its constant relationship with the external environment (eating, oxygen availability, communicating, defending against infection, etc.) [18].

Initially, it was thought that it was the humoral immune response to Porphyromonas gingivalis, a prominent oral pathogen linked to gingivitis and periodontal disease (PD), which was involved in the onset of RA [19]. Peptidyl-arginine deiminase (PAD) is produced by $P$. gingivalis and can citrullinated antigens/proteins in patients with RA. In RA, citrullination is a physiological process in many tissues and can lead to immune response [20]. Given that PAD can citrullinated antigens, a link was loosely established that increased levels of PAD producing $P$. gingivalis in the oral cavity play a part in activating $\mathrm{T}$ cells, which in turn, will provide antigen-specific help to B cells to generate the ACPAs in RA patients [21].

Aggregatibacter actinomycetemcomitans and Anaeroglobus geminatus were later added to the list of microbes associated with increased disease severity in RA. A. actinomycetemcomitans is involved in hypercitrullination in host neutrophils, inducing an immune reaction in RA patients [21], and A. geminatus, was correlated with ACPA/rheumatoid factor (RF) presence in RA patients with PD [22].

Another organism, capable of producing large amounts of citrulline, Cryptobacterium curtum, emerged as a robust discriminant of the oral microbiome in individuals with RA without PD [23]. Further sequencing determined that dental and salivary samples of patients at high risk for developing RA showed enrichment of Lactobacillus salivarius, Atopobium spp., and C. curtum [24]. L. salivarius is prominent in very active cases of RA and was positively correlated with increasing immunoglobulin G (IgG), C-reactive protein (CRP), and erythrocyte sedimentation rate (ESR) levels [25]. Together, these studies showed the importance of oral microbial species in the pathogenesis of RA.

The variations in the gut microbiome in RA patients have also been implicated in RA pathogenesis [5]. The healthy gut microbiome is characterized by three major phyla: Firmicutes, Bacteroidetes, and Actinobacteria [26]. This microbiome serves as a functional expansion of host genomes and is estimated to harbor 50-100 folds more genes than the host [27]. These extra genes add various types of enzymes which are not encoded by the host and play a critical role in facilitating and regulating host metabolism and physiology [28]. In healthy adults, fragments of normal intestinal bacteria are found in circulating blood leukocytes in the spleen. In patients with inflammatory arthritis, similar degradation products of bacterial cell walls and nucleic acids were detected in the inflamed joints [29]. In individuals at risk for RA, the arthritogenic bacterial antigens would pass from the intestines to the joints [30]. This suggests that individuals with genetic susceptibility for RA are prone to harbor intestinal microbiota containing specifically arthritogenic bacterial species or stains leading to aberrant immune responses and chronic inflammation [31]. 
Prevotella copri (Pc) was discovered in new-onset, untreated RA (NORA) patients, as an intestinal microbe correlated with disease activity [32]. Increases in Pc abundance correlated with a reduction in Bacteroides and a loss of beneficial microbes in NORA patients [27]. The prevalence of Pc in chronic RA (CRA) patients being treated and exhibiting reduced disease activity, was similar to healthy subjects [33]. One possibility is Pc fails to thrive when there is less inflammation due to a lack of terminal electron acceptors [33]. Despite the links between the intestinal microbiome and RA development, it remains unclear whether these states of oral and gut dysbiosis represent a causal-etiological factor or a secondary effect of local and systemic inflammation.

\section{Role of oral and gut microbiome in CD}

The microbiome's role in CD has been studied extensively due to gluten's effect on the intestinal membrane [33]. However, little is known regarding the association between dysbiosis and gluten-specific T-cell response relevance in CD [34]. It has been shown that members of the phylum Proteobacteria are more abundant and those of the Firmicutes and Actinobacteria phyla are less abundant in active CD than in GFD patients or controls [34].

Caminero et al. found that bacterial colonization produced distinct gluten-degradation in the mouse small intestine [33]. Pseudomonas aeruginosa, an opportunistic pathogen from CD patients, exhibited elastase activity which allows producing of shorter gluten peptides [33]. These peptides often highly immunogenic effectively translocated the mouse intestinal barrier [33]. However, mice incubated with P. aeruginos $a$ and Lactobacillus spp. showed reduced major immunogenic peptides, suggesting that immunogenic peptides generated by $P$. aeruginosa can be degraded to non-immunogenic peptides in the presence of Lactobacillus spp. [33].

In patients with $\mathrm{CD}$ and patients with refractory $\mathrm{CD}(\mathrm{RCD}, \mathrm{CD}$ unresponsive/resistant to at least 12 months of treatment with GFD), levels of these bacteria fluctuated [35]. Significant differences between patients with CD and RCD groups in Bacteroidetes (CD>RCD), Actinobacteria (CD<RCD), and Fusobacteria (CD $>$ RCD) were found. The oral microbiome of patients with RCD was significantly different from the one in patients with CD. As CD progresses to RCD, oral microbial colonization patterns change [35]. These changes offer new opportunities for screening, diagnosis, and potential evaluation of the risks of a CD patient transitioning to RCD [35]. Nonetheless, additional research is required to confirm this presumption.

\section{Probiotics as treatment}

The Food and Agriculture Organization (FAO)/World Health Organization (WHO), defines a probiotic as a "live microorganism, which when administered in adequate amount confers a health benefit on the host" [36]. The benefits of probiotics on host gut health can be manifested through (i) production of inhibitory substances against pathogens, (ii) blockage of adhesion sites, (iii) competition for nutrients, (iv) degradation of toxin receptors, and (v) regulation of immunity [37]. Over the past five years, new research has shed a light on novel probiotic strains that could be useful for the treatment of RA and CD [38-45].

\section{Probiotics and rheumatoid arthritis}

Since 2015, several novel probiotics have been shown to modulate RA disease severity by altering gut microbe composition in mouse/rat models and human subjects [38-41]. In human subjects with RA, supplementation of Lactobacillus acidophilus, Lactobacillus casei, and Bifidobacterium bifidum for eight weeks exhibited an improvement in disease activity score, a significant decrease in serum insulin, and high sensitivity C- reactive protein (hsCRP) levels [39]. The patients showed statistically significant improvement of total and low-density lipoprotein (LDL) cholesterol levels. L. casei was shown to lower pro-inflammatory cytokines interleukin-12 (IL-12) and tumor necrosis factor- $\alpha$ (TNF- $\alpha$ ) levels with reduced swollen joints after treatment [39]. Intervention with Bifidobacterium animalis subspecies (subsp.) lactis GCL2505 (BlaG) resulted in reduced severity of diseased joints [40]. Treatment with BlaG has been documented to increase levels of Lactobacillus and Bifidobacterium, which were positively correlated to the production of protective short-chain fatty acids (SCFAs) [40].

A study using a rat model of arthritis showed a reduction of TNF- $\alpha$ levels with a decrease in disease activity after oral treatment with Bacillus coagulans and inulin [41]. The reduction in TNF- $\alpha$ was observed to be similar to the anti-inflammatory effects of indomethacin (NSAID) [41]. Prevotella histicola isolated from the human gut showed a reduced incidence of disease with delayed onset in HLA-DQ8 RA induced mice [40]. The treatment has reduced cellular and humoral immunity by lowering autoantibodies and decreasing antigenspecific T-cell proliferation [40]. The decrease in pro-inflammatory cytokines and increase in regulatory cells in the gut led to an increase in tight junction proteins and maintenance of gut epithelial integrity [40]. This study suggests $P$. histicola would be a useful probiotic treatment for RA, especially since the strain is endogenous to the human gut [40]. This possibility would allow for fewer side effects, and the capability to treat patients with targeted antibiotics if side effects do arise [40].

\section{Probiotics and celiac disease}

A concoction of Streptococcus thermophilus, Lactobacillus plantarum, L. acidophilus, L. casei, L. delbrueckii 
subsp. bulgaricus, Bifidobacterium breve, B. longum, B. infantis, L. salivarus spp. subsp. salicinius, known as VSL\#3, is the treatment for irritable bowel syndrome (IBS), and ileal pouch [42]. In 2006, Angelis et al. showed how VSL \#3 could be useful in treating celiac sprue (CS). The epitopes involved in stimulating an excessive immune response in CS contain a large amount of proline (Pro) residues [46]. As an amino acid, Pro is unique due to its cyclic structure. This conformation establishes many restrictions regarding the structural aspects of peptides and proteins, resulting in increased resistance to hydrolysis [46]. The mass spectrometry showed an almost complete deterioration of gliadins during long-time fermentation of wheat flour by VSL\#3 in mouse models [43]. However, VSL \#3 was unable to hydrolyze gliadins when each of its component strains were tested separately, this implies that no single probiotic strain in VSL \#3 contained all the required peptidases needed to break down gliadins [43].

Several strains alleviate symptoms in patients with active CD: Bifidobacterium infantis Natren Life Strain super strain (NLS-SS) [43], Bifidobacterium breve strains (BR03 and B632) [44,45], and a cocktail of five strains of lactic acid bacteria and Bifidobacteria: L. casei LMG 101/37 P-17504, L. plantarum CECT 4528, B. animalis subsp. lactis Bi1 LMGP-17502, Bifidobacterium breve Bbr8 LMG P-17501, and Bifidobacterium breve Bl10 LMG P-17500 [47]. Pinto-Sanchez et al. tested the impact of Bifidobacterium infantis NLS-SS by evaluating Paneth cells, macrophage counts, and human-defensin 5 (HD5) expression in duodenal biopsies of CD patients on a GFD [43]. Their results revealed that patients treated with NLS-SS experienced a decline in HD5 expression and Paneth cell counts [48]. Klemenak et al. explored the action of two Bifidobacterium breve strains (BR03 and B632) on serum IL-10 and TNF- $\alpha$ levels in 49 children with CD on GFD, showing lower levels of TNF- $\alpha$ after daily use for three months [45]. Primec et al. carried out a double-blind placebocontrolled study enrolling $40 \mathrm{CD}$ and 16 healthy children [44]. In another study, children with CD were randomized to receive either a placebo or a cocktail of two Bifidobacterium breve strains BR03 (DSM 16604) and B632 (DSM 24706) for three months [49]. This probiotic combination was able to alter the production of acetic acid and total short-chain fatty acids and ultimately played a role in stimulating the restoration of the microbiome [44].

\section{Conclusions}

The microbiome is an essential immune modulator, and its dysbiosis is thought to result in a negative impact on health. The research has shed light on a new role of the microbiome in RA and CD, and the intricate relationship between its make-up and the presentation of clinical symptoms. While many crucial issues remain to be identified, some aspects are now evident: (a) patients with RA and CD have alterations in the composition of both their oral and gut microbiome, (b) these alterations are associated with disease activity, and (c) the use of probiotics (in adjunct with disease-centered treatment) have shown to be effective in improving symptom severity by changing the microbial composition of the oral or gut microbiome. Together, these studies strongly indicate an association between dysbiosis of the gut and oral microbiomes that negatively influence the gut-joint axis. These studies support our anecdotal clinical encounters suggesting RA patients with CD have increased rheumatic disease severity (data not shown). Future studies on the pathological role of the microbial strains implicated in CD and RA and the impact of probiotics in reducing their levels are needed to better understand this link.

\section{Additional Information \\ Disclosures}

Conflicts of interest: In compliance with the ICMJE uniform disclosure form, all authors declare the following: Payment/services info: All authors have declared that no financial support was received from any organization for the submitted work. Financial relationships: All authors have declared that they have no financial relationships at present or within the previous three years with any organizations that might have an interest in the submitted work. Other relationships: All authors have declared that there are no other relationships or activities that could appear to have influenced the submitted work.

\section{References}

1. Guo Q, Wang Y, Xu D, Nossent J, Pavlos NJ, Xu J: Rheumatoid arthritis: pathological mechanisms and modern pharmacologic therapies. Bone Res. 2018, 6:15. 10.1038/s41413-018-0016-9

2. Becker J, Winthrop KL: Update on rheumatic manifestations of infectious diseases . Curr Opin Rheumatol. 2010, 22:72-77. 10.1097/BOR.0b013e328333b9f5

3. Gregersen PK, Silver J, Winchester RJ: The shared epitope hypothesis. An approach to understanding the molecular genetics of susceptibility to rheumatoid arthritis. Arthritis Rheum. 1987, 30:1205-1213. 10.1002/art.1780301102

4. Brusca SB, Abramson SB, Scher JU: Microbiome and mucosal inflammation as extra-articular triggers for rheumatoid arthritis and autoimmunity. Curr Opin Rheumatol. 2014, 26:101-107. 10.1097/BOR.0000000000000008

5. Scher JU, Abramson SB: The microbiome and rheumatoid arthritis . Nat Rev Rheumatol. 2011, 7:569-578. 10.1038/nrrheum.2011.121

6. Wasserman AM: Diagnosis and management of rheumatoid arthritis . Am Fam Physician. 2011, 84:12451252.

7. Lerner A, Matthias T: Rheumatoid arthritis-celiac disease relationship: joints get that gut feeling . Autoimmun Rev. 2015, 14:1038-1047. 10.1016/j.autrev.2015.07.007 
8. Sharma N, Bhatia S, Chunduri V, et al.: Pathogenesis of celiac disease and other gluten related disorders in wheat and strategies for mitigating them. Front Nutr. 2020, 7:6. 10.3389/fnut.2020.00006

9. Wang F, Liu Y, Lou S, et al.: A review on the clinicopathological features and management of the celiac disease. Gastroenterol Hepatol Lett. 2020, 2:6-10.

10. Shor DB, Orbach H, Boaz M, et al.: Gastrointestinal-associated autoantibodies in different autoimmune diseases. Am J Clin Exp Immunol. 2012, 1:49-55.

11. Stoll ML, Patel AS, Christadoss ML, Punaro M, Olsen NJ: IgA transglutaminase levels in children with juvenile idiopathic arthritis. Ann Paediatr Rheumatol. 2012, 1:31-35.

12. Peschken C, Hitchon C: Rising prevalence of systemic autoimmune rheumatic disease: increased awareness, increased disease or increased survival?. Arthritis Res Ther. 2012, 14:A20. 10.1186/ar3954

13. Hunter TM, Boytsov NN, Zhang X, Schroeder K, Michaud K, Araujo AB: Prevalence of rheumatoid arthritis in the United States adult population in healthcare claims databases, 2004-2014. Rheumatol Int. 2017, 37:1551-1557. 10.1007/s00296-017-3726-1

14. Ciacci C, Cirillo M, Sollazzo R, Savino G, Sabbatini F, Mazzacca G: Gender and clinical presentation in adult celiac disease. Scand J Gastroenterol. 1995, 30:1077-1081. 10.3109/00365529509101610

15. van Vollenhoven RF: Sex differences in rheumatoid arthritis: more than meets the eye . BMC Med. 2009, 7:12. 10.1186/1741-7015-7-12

16. Voigt LF, Koepsell TD, Nelson JL, Dugowson CE, Daling JR: Smoking, obesity, alcohol consumption, and risk of rheumatoid arthritis. Epidemiology. 1994, 5:525-532.

17. Aas JA, Paster BJ, Stokes LN, Olsen I, Dewhirst FE: Defining the normal bacterial flora of the oral cavity . J Clin Microbiol. 2005, 43:5721-5732.

18. Parahitiyawa NB, Scully C, Leung WK, Yam WC, Jin LJ, Samaranayake LP: Exploring the oral bacterial flora: current status and future directions. Oral Dis. 2010, 16:136-145. 10.1111/j.1601-0825.2009.01607.x

19. Rosenstein ED, Greenwald RA, Kushner LJ, Weissmann G: Hypothesis: the humoral immune response to oral bacteria provides a stimulus for the development of rheumatoid arthritis. Inflammation. 2004, 28:311-318. 10.1007/s10753-004-6641-z

20. Sakkas LI, Daoussis D, Liossis SN, Bogdanos DP: The infectious basis of ACPA-positive rheumatoid arthritis . Front Microbiol. 2017, 8:1853. 10.3389/fmicb.2017.01853

21. Konig MF, Abusleme L, Reinholdt J, et al.: Aggregatibacter actinomycetemcomitans-induced hypercitrullination links periodontal infection to autoimmunity in rheumatoid arthritis. Sci Transl Med. 2016, 8:369ra176. 10.1126/scitranslmed.aaj1921

22. Scher JU, Ubeda C, Equinda M, et al.: Periodontal disease and the oral microbiota in new-onset rheumatoid arthritis. Arthritis Rheum. 2012, 64:3083-3094. 10.1002/art.34539

23. Lopez-Oliva I, Paropkari AD, Saraswat S, et al.: Dysbiotic subgingival microbial communities in periodontally healthy patients with rheumatoid arthritis. Arthritis Rheumatol. 2018, 70:1008-1013. 10.1002/art.40485

24. Zhang X, Zhang D, Jia H, et al.: The oral and gut microbiomes are perturbed in rheumatoid arthritis and partly normalized after treatment. Nat Med. 2015, 21:895-905. 10.1038/nm.3914

25. Tong Y, Zheng L, Qing P, et al.: Oral microbiota perturbations are linked to high risk for rheumatoid arthritis. Front Cell Infect Microbiol. 2019, 9:475. 10.3389/fcimb.2019.00475

26. Rinninella E, Raoul P, Cintoni M, Franceschi F, Miggiano GAD, Gasbarrini A, Mele MC: What is the healthy gut microbiota composition? A changing ecosystem across age, environment, diet, and diseases. Microorganisms. 2019, 7:14. 10.3390/microorganisms7010014

27. Eckburg PB, Bik EM, Bernstein CN, et al.: Diversity of the human intestinal microbial flora. Science. 2005, 308:1635-1638. 10.1126/science.1110591

28. Hooper LV, Wong MH, Thelin A, Hansson L, Falk PG, Gordon JI: Molecular analysis of commensal hostmicrobial relationships in the intestine. Science. 2001, 291:881-884. 10.1126/science.291.5505.881

29. Schrijver IA, Melief MJ, Tak PP, Hazenberg MP, Laman JD: Antigen-presenting cells containing bacterial peptidoglycan in synovial tissues of rheumatoid arthritis patients coexpress costimulatory molecules and cytokines. Arthritis Rheumatol. 2001, 43:2160-2168. 10.1002/1529-0131(200010)43:10<2160::AIDANR3>3.0.CO;2-T

30. Vaahtovuo J, Munukka E, Korkeamäki M, Luukkainen R, Toivanen P: Fecal microbiota in early rheumatoid arthritis. J Rheumatol. 2008, 35:1500-1505.

31. Zoetendal EG, Akkermans ADL, Akkermans-van Vliet WM, de Visser GM, de Vos WM: The host genotype affects the bacterial community in the human gastrointestinal tract. Microb Ecol Health Dis. 2001, 13:129134. 10.1080/089106001750462669

32. Scher JU, Sczesnak A, Longman RS, et al.: Expansion of intestinal prevotella copri correlates with enhanced susceptibility to arthritis. Elife. 2013, 2:e01202. 10.7554/eLife.01202.001

33. Caminero A, Galipeau HJ, McCarville JL, et al.: Duodenal bacteria from patients with celiac disease and healthy subjects distinctly affect gluten breakdown and immunogenicity. Gastroenterology. 2016, 151:670683. 10.1053/j.gastro.2016.06.041

34. D'Argenio V, Casaburi G, Precone V, et al.: Metagenomics reveals dysbiosis and a potentially pathogenic N. flavescens strain in duodenum of adult celiac patients. Am J Gastroenterol. 2016, 111:879-890. 10.1038/ajg.2016.95

35. Tian N, Faller L, Leffler DA, et al.: Salivary gluten degradation and oral microbial profiles in healthy individuals and celiac disease patients. Appl Environ Microbiol. 2017, 83:e03330-16. 10.1128/AEM.03330-16

36. Guidelines for the evaluation of probiotics in food . (2002). Accessed: January 12, 2021: https://www.who.int/foodsafety/fs_management/en/probiotic_guidelines.pdf.

37. Vanderpool C, Yan F, Polk DB: Mechanisms of probiotic action: implications for therapeutic applications in inflammatory bowel diseases. Inflamm Bowel Dis. 2008, 14:1585-1596. 10.1002/ibd.20525

38. Zamani B, Golkar HR, Farshbaf S, et al.: Clinical and metabolic response to probiotic supplementation in patients with rheumatoid arthritis: a randomized, double-blind, placebo-controlled trial. Int J Rheum Dis. 2016, 19:869-879. 10.1111/1756-185X.12888

39. Aoki R, Kamikado K, Suda W, et al.: A proliferative probiotic Bifidobacterium strain in the gut ameliorates 
progression of metabolic disorders via microbiota modulation and acetate elevation. Sci Rep. 2017, 7:43522. 10.1038/srep43522

40. Marietta EV, Murray JA, Luckey DH, et al.: Suppression of inflammatory arthritis by human gut-derived Prevotella histicola in humanized mice. Arthritis Rheumatol. 2016, 68:2878-2888. 10.1002/art.39785

41. Abhari K, Shekarforoush SS, Hosseinzadeh S, Nazifi S, Sajedianfard J, Eskandari MH: The effects of orally administered Bacillus coagulans and inulin on prevention and progression of rheumatoid arthritis in rats. Food Nutr Res. 2016, 60:30876. 10.3402/fnr.v60.30876

42. de Sousa Moraes LF, Grzeskowiak LM, de Sales Teixeira TF, do Carmo Gouveia Peluzio M: Intestinal microbiota and probiotics in celiac disease. Clin Microbiol Rev. 2014, 27:482-489. 10.1128/CMR.00106-13

43. Pinto-Sánchez MI, Smecuol EC, Temprano MP, et al.: Bifidobacterium infantis NLS super strain reduces the expression of $\alpha$-defensin-5, a marker of innate immunity, in the mucosa of active celiac disease patients. J Clin Gastroenterol. 2017, 51:814-817. 10.1097/MCG.0000000000000687

44. Primec M, Klemenak M, Di Gioia D, et al.: Clinical intervention using Bifidobacterium strains in celiac disease children reveals novel microbial modulators of TNF- $\alpha$ and short-chain fatty acids. Clin Nutr. 2019, 38:1373-1381. 10.1016/j.clnu.2018.06.931

45. Klemenak M, Dolinšek J, Langerholc T, Di Gioia D, Mičetić-Turk D: Administration of Bifidobacterium breve decreases the production of TNF- $\alpha$ in children with celiac disease. Dig Dis Sci. 2015, 60:3386-3392. 10.1007/s10620-015-3769-7

46. De Angelis M, Rizzello CG, Fasano A, et al.: VSL\#3 probiotic preparation has the capacity to hydrolyze gliadin polypeptides responsible for Celiac Sprue. Biochim Biophys Acta. 2006, 1762:80-93. 10.1016/j.bbadis.2005.09.008

47. Francavilla R, Piccolo M, Francavilla A, et al.: Clinical and microbiological effect of a multispecies probiotic supplementation in celiac patients with persistent IBS-type symptoms: a randomized, double-blind, placebo-controlled, multicenter trial. J Clin Gastroenterol. 2019, 53:117-125. 10.1097/MCG.0000000000001023

48. Cristofori F, Indrio F, Miniello VL, De Angelis M, Francavilla R: Probiotics in celiac disease. Nutrients. 2018, 10:1824. 10.3390/nu10121824

49. Bozzi Cionci N, Baffoni L, Gaggì F, Di Gioia D: Therapeutic microbiology: the role of Bifidobacterium breve as food supplement for the prevention/treatment of paediatric diseases. Nutrients. 2018, 10:1723. 10.3390/nu10111723 